\title{
Photon echoes in optically dense media
}

\author{
Sergey A. Moiseev, ${ }^{1}$ Mahmood Sabooni, ${ }^{2,3}$ and Ravil V. Urmancheev ${ }^{1}$ \\ ${ }^{1}$ Kazan Quantum Center, Kazan National Research Technical University named after A. N. Tupolev-KAI, 10 K. Marx, Kazan 420111, Russia \\ ${ }^{2}$ Institute for Quantum Computing, Department of Physics and Astronomy, University of Waterloo, Waterloo, Ontario N2L 3G1, Canada \\ ${ }^{3}$ Department of Physics, University of Tehran, 14399-55961 Tehran, Iran
}

(Received 25 April 2019; published 24 January 2020)

\begin{abstract}
Coherent nonlinear multipulse processes, nonlinear waves, and echo effects in resonant media are the topical problems of modern optics and important tools of coherent spectroscopy and quantum information science. We generalize the McCall-Hahn area theorem to the formation of an arbitrary photon echo generated during the multipulse excitation of the optically dense resonant media. The derived theorem made it possible to reveal the nonlinear mechanism of generation and evolution of the photon echo signals inside the media after a twopulse excitation. We find that a series of self-reviving echo signals with a total area of $2 \pi$ or $0 \pi$ is excited and propagates in the media depth, with each pulse having an individual area less than $\pi$. The resulting echo pulse train is an alternative to the well-known soliton or breather. The developed pulse-area approach paves the way for more precise coherent spectroscopy, studies of different photon echo signals, and quantum control of light pulses in the optically dense media.
\end{abstract}

DOI: 10.1103/PhysRevResearch.2.012026

Studies of coherent multipulse nonlinear effects such as photon echo and four-wave mixing open wide opportunities for understating light-atom interactions, fundamental processes of nonlinear and quantum optics, provide powerful techniques for spectroscopic investigation of atoms and molecules, and are considered as a principal tool for implementation of basic processes in practical quantum information science [1-5]. Herein, the photon echo technique [6,7] attracts special, long-lasting attention in coherent spectroscopy [7] and light pulse storage [8-12]. Recently, the photon echo in optically dense media opened promising opportunities for quantum storage of a large number of light pulses [13-17] and quantum processing [18] that determined a steady interest and elaboration of numerous protocols of photon-echobased quantum memories [14,19-24], which are important for the creation of quantum repeaters [25], microwave quantum memory [26,27], etc.

The study of the properties of two- and three-pulse photon echoes in optically dense media is the main task in the development of multipulse spectroscopy and photon echo quantum memory schemes in such media. The most general theoretical description of the coherent resonant interaction of multipulse light fields with resonant atoms can be provided by the pulse area theorem [28-35]. In early works on the two-pulse (primary) photon echo, it was found that the initial excitation could result in the generation of multiple echo signals $[30,36]$ followed by a long-term investigation of the underlying mechanism [29,30,37-44]. Quite early an

Published by the American Physical Society under the terms of the Creative Commons Attribution 4.0 International license. Further distribution of this work must maintain attribution to the author(s) and the published article's title, journal citation, and DOI. analytic solution for total area of all the echoes was obtained $[30,36,39]$, which proved that the total pulse area can tend asymptotically toward $2 \pi$ in the media depth if the initial pulse area of two exciting laser pulses exceeds $\pi$. However, this solution does not allow one to describe the behavior of each individual echo pulse.

A previously acquired solution for the primary echo pulse area predicted that the echo pulse area never exceeds $\pi$ and generally decays in the depth of the media [38]. This finding again stressed the ambiguity of the known physical picture behind the formation of the total nonlinear response to the multipulse excitation. In recent years the stakes were raised by the demand for an efficient optical solid-state quantum memory and the noted interest in coherent multipulse interactions in the optically dense media.

In this Rapid Communication we find an analytical solution of the photon echo pulse area theorem posed in Refs. [29,36,37] in 1971. By analyzing the solution we discover the mechanism of self-induced transparency [28] for two- and many-pulse excitation of the atomic media leading to the formation of many echo pulses. To do that we find the general analytic solution for the pulse area of an arbitrary secondary photon echo signal. The found solutions show that the echo signals are excited coherently one after another in a certain area of the medium and then disappear, generating new echo signals and creating a self-reviving echo sequence. We show that depending on the input pulse areas this echo pulse train forms a multipulse analog to the well-known single-pulse $2 \pi$ optical soliton or a $0 \pi$ optical breather despite each individual echo pulse area never exceeding $\pi$. Herein, by using the highly nonlinear nature of the light-atom interaction we can control the total response of the media. Being near the threshold, when the incoming area of the second pulse is close to $\pi$, and by slightly changing it to being $<\pi$ or $>\pi$, one can initiate a huge change in the outcome from an optical soliton 
to an optical breather, respectively. This also demonstrates the potential of the pulse area approach for coherent spectroscopy of the optically dense media.

First we reproduce the McCall-Hahn area theorem and derive the general equation for the pulse area of an arbitrary echo pulse starting with the usual reduced set of MaxwellBloch equations [30] for the light field and atomic system:

$$
\begin{aligned}
{\left[\partial_{z}+c^{-1} \partial_{t}\right] \Omega } & =i \frac{\mu}{2}\langle P\rangle, \\
\partial_{t} u & =-\Delta v-\gamma u, \\
\partial_{t} v & =\Delta u-\gamma v+\Omega w, \\
\partial_{t} w & =-\Omega v,
\end{aligned}
$$

where $\vec{r}=\vec{r}(t, z, \Delta)=(u, v, w)^{T}$ is the Bloch vector, each component depending on time $t$, spatial coordinate $z$, and atomic detuning $\Delta ; P=u-i v$ is the atomic polarization; electric field $E(t, z)=\varepsilon(t, z) \exp [i(k z-\omega t)]+$ c.c. is described by a complex light field envelope $\varepsilon(t, z)$ with corresponding Rabi frequency $\Omega(t, z)=(2 d / \hbar) \varepsilon(t, z) ; \mu=$ $4 \pi N d^{2} \omega / \hbar c ; \gamma=1 / T_{2}, T_{2}$ is the coherence lifetime of the atomic transition; and $\langle\ldots\rangle \equiv \int_{-\infty}^{\infty} G(\Delta) \ldots d \Delta$ is the averaging over the inhomogeneous broadening. From now on for simplicity, we do not denote the existing dependence on $z$ in atomic and field variables $\vec{r}$ and $\Omega$.

We transfer to the pulse area $\theta=\int_{-\infty}^{\infty} d t \Omega(t)$ and follow [28,31] to find that incoming pulse areas $\theta_{1}, \theta_{2}$ satisfy the well-known pulse area theorem

$$
\partial_{z} \theta=\frac{1}{2} \alpha w_{0}(z) \sin \theta(z),
$$

where $w_{0}$ is the initial inversion of the atomic system and $\alpha$ is the resonant absorption coefficient [30]. The first pulse propagates in the undisturbed media, with $w_{0}=-1$, and partially inverts for the second pulse, so $w_{0}=-\cos \theta_{1}$. Substituting $w_{0}$ into Eq. (2) we get the well-known solutions [30]

$$
\begin{aligned}
& \theta_{1}(z)=2 \arctan \left[e^{-\alpha z / 2} \tan \frac{\theta_{1}(0)}{2}\right], \\
& \theta_{2}(z)=2 \arctan \left[\kappa \operatorname{sech}\left(\beta-\frac{\alpha}{2} z\right)\right],
\end{aligned}
$$

where $\beta=\ln \left\{\tan \left[\frac{\theta_{1}(0)}{2}\right]\right\}$ and $\kappa=\tan \left[\frac{\theta_{2}(0)}{2}\right] / \sin \left[\theta_{1}(0)\right]$.

Equations (2) and (3) can be used to find the total area of all excited photon echoes $[30,36,37,39]$ :

$\theta_{\Sigma e}(z)=2 \arctan \left[e^{-\alpha z / 2} \tan \frac{\theta_{1}(0)+\theta_{2}(0)}{2}\right]-\theta_{2}(z)-\theta_{1}(z)$.

This solution predicts that if $\theta_{2}(0)<\pi, \theta_{1}(0)+\theta_{2}(0)>\pi$, the total area of all echo pulses asymptotically tends to $2 \pi$ [36]. It leaves however a lot of uncertainty about the mechanism and physics of the photon echo generation, since any information about the particular photon echo signals remains hidden. How exactly do different echoes combine into the $2 \pi$ pulse area? What is the contribution of an individual echo? Moreover, if input pulse areas $\theta_{1}(0)<\pi / 2, \theta_{2}(0)>$ $\pi$, Eq. (4) predicts the sum of all echoes to be 0 . What happens with the different echo signals in this case, and does that mean that there will be no echoes? To answer all these

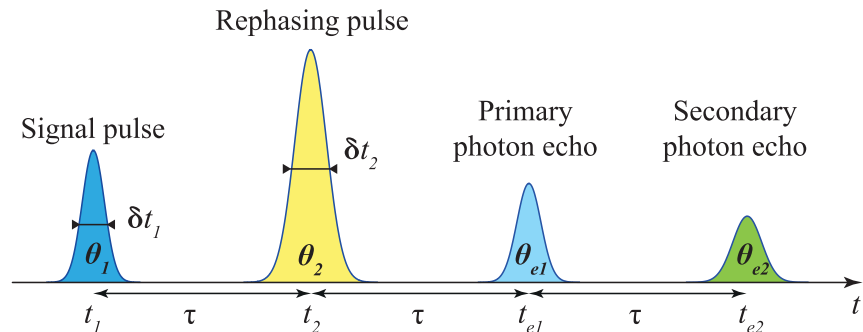

FIG. 1. Time delays and intervals between the pulses involved in the formation of primary and secondary echoes. The pulses are well separated and resonant with atomic transition with large inhomogeneous broadening and long coherence lifetime: $1 / \Delta_{\text {inh }} \ll$ $\delta t_{1,2} \ll \tau \ll T_{2}$.

questions, we have to analyze the generation of each echo signal individually.

To find the area theorem for an arbitrary individual photon echo signal we integrate the first of Eqs. (1) over time around the time of echo emission $t_{e}$, from $t_{0}=t_{e}-\tau / 2$ to $t_{1}=t_{e}+$ $\tau / 2$, where $\tau$ is the delay between the pulses (see Fig. 1). We should also clarify the timescales assumed for the following derivation. First, we assume nonoverlapping pulses $\tau \gg \delta t_{1,2}$ with pulse duration being much smaller than coherence time $\delta t_{i} \ll T_{2}, i=1,2, e 1, \ldots$, to neglect the relaxation during the pulses. Second, inhomogeneous broadening of the atomic system is much larger that the pulse spectrum $\Delta_{i n}>1 / \delta t_{1,2}$. Third, for simplicity we consider a solid-state system, meaning $T_{1} \gg T_{2}$, and thus we can neglect the population decay between the pulses. In short, $1 / \Delta_{\text {in }}<\delta t_{1,2} \ll \tau \lesssim T_{2}$.

The expressions under the integrals, $P_{0}(z, \Delta)$ and $w(t, z, \Delta)$, are complex expressions consisting of several oscillating components. However, most of these components will give 0 after averaging over $\Delta$ in Eq. (1). To find the proper expression for the echo area we need to only take into account the phasing components of polarization and inversion that contribute to the echo formation. The details of the integration and equation handling can be found in the Supplemental Material [46].

As a result we obtain the general equation for an arbitrary echo pulse area:

$$
\partial_{z} \theta(z)=\frac{1}{2} \alpha\left[2 v_{0}(z) \cos ^{2} \frac{\theta(z)}{2}+w_{0}(z) \sin \theta(z)\right],
$$

where $w_{0}(z)$ and $v_{0}(z)$ are the initial values $\left(t=t_{e}-\tau / 2\right)$ of the Bloch vector resonance components with $\Delta=0$ which only give nonzero response in the field equation in Eq. (1). After transition to $\eta=\tan \frac{\theta(z)}{2}$ we get a linear equation $\partial_{z} \eta(z)=$ $\frac{\alpha}{2}\left[v_{0}(z)+w_{0}(z) \eta(z)\right]$ with a clear solution.

Equation (5) describes the pulse area of a chosen echo signal given the phasing coherence $v_{0}$ in the presence of spectral uniform inversion $w_{0}$ and Eq. (5) comes down to finding $v_{0}(z)$ and $w_{0}(z)$ for each echo signal. In the Supplemental Material [46] we describe the algorithm that allows to find the $v_{0}, w_{0}$ for an arbitrary echo. But whatever they may be, we note that $|\theta|$ never exceeds $\pi$. Below we investigate the analytic solutions for the pulse areas of all the echo signals.

For the primary echo we have $\vec{r}(t)=U(t-$ $\tau) T\left(\theta_{2}\right) U(\tau) T\left(\theta_{1}\right) \vec{r}(0), t_{0}=3 \tau / 2$, and the correct phasing 
components of $\tilde{v}_{0}(3 \tau / 2), \tilde{w}_{0}(3 \tau / 2)[38,45]$ :

$$
\begin{gathered}
v_{0}(3 \tau / 2, z)=\Gamma_{\tau}^{2} \sin \theta_{1}(z) \sin ^{2} \frac{\theta_{2}(z)}{2}, \\
\tilde{w}_{0}(3 \tau / 2, z)=-\cos \theta_{1}(z) \cos \theta_{2}(z),
\end{gathered}
$$

where $\Gamma_{\tau}=e^{-\gamma \tau}$ is the relaxation term. An equation corresponding to Eq. (5) gives the primary photon echo pulse area:

$$
\theta_{e 1}(z)=2 \arctan \left[\Gamma_{\tau}^{2} \sin \theta_{1}(0) \sin ^{2} \frac{\theta_{2}(z)}{2} \sinh \frac{\alpha z}{2}\right] .
$$

After the incoming pulses and the primary echo pulse we have $\vec{r}(t)=U(t-2 \tau) T\left(\theta_{e 1}\right) U(\tau) T\left(\theta_{2}\right) U(\tau) T\left(\theta_{1}\right) \vec{r}(0), t_{0}=$ $5 \tau / 2$, and the phasing components $v_{0}(5 \tau / 2, z), w_{0}(5 \tau / 2, z)$ are

$$
\begin{aligned}
v_{0}= & v_{01}+v_{02} \\
= & \frac{1}{2} \Gamma_{\tau}^{2} \sin \theta_{1}(z) \sin \theta_{e 1}(z) \sin \theta_{2}(z) \\
& +\Gamma_{\tau}^{2} \cos \theta_{1}(z) \sin ^{2} \frac{\theta_{e 1}(z)}{2} \sin \theta_{2}(z), \\
w_{0}= & w_{01}+w_{02} \\
= & -\Gamma_{\tau}^{2} \sin \theta_{1}(z) \sin ^{2} \frac{\theta_{2}(z)}{2} \sin \theta_{e 1}(z) \\
& -\cos \theta_{1}(z) \cos \theta_{2}(z) \cos \theta_{e 1}(z) .
\end{aligned}
$$

The first terms in both equations $v_{01}(z)=$ $\frac{1}{2} \Gamma_{\tau}^{2} \sin \theta_{1} \sin \theta_{2} \sin \theta_{e 1}$ and $w_{01}(z)=-\Gamma_{\tau}^{2} \sin \theta_{1} \sin ^{2} \frac{\theta_{2}}{2} \sin \theta_{e 1}$ are proportional to $\sin \theta_{1}(z)$ and vanish when the first pulse is absorbed. They are responsible for the stimulated photon echo generated by incoming pulses and the primary echo pulse. The other two components $v_{02}(z)=\Gamma_{\tau}^{2} \cos \theta_{1} \sin \theta_{2} \sin ^{2} \frac{\theta_{e 1}}{2}$ and $w_{02}(z)=-\cos \theta_{1} \cos \theta_{2} \cos \theta_{e 1}$ are proportional to $\cos \theta_{1}$ and correspond to the secondary two-pulse photon echo created by the second pulse and the primary echo pulse.

Analysis of the successive echoes follows the same procedure but requires more calculations since $v_{0}$ and $w_{0}$ have more terms with each step. In the Supplemental Material [46] we introduce the phasing polarization and inversion components for the third and the fourth echoes and discuss the physical meaning of different contributions. It is obvious that the described procedure can be applied for the case with comparable transverse and longitudinal relaxations and for other light-atom equations.

We will now proceed to clarify the mechanism of the total $2 \pi$ pulse area formation when $\theta_{1}(0)+\theta_{2}(0)>\pi$. Figure 2 shows the spatial behavior of the area of incoming pulses and echo pulses, and the total area depending on the optical density of the medium for $\theta_{1}(0)=0.1 \pi, \theta_{2}(0)=0.999 \pi$. We see that incoming pulses excite primary and secondary echoes that in turn excite subsequent echoes. Each echo pulse is born, propagates, and eventually dies out within a finite spatial interval. However the total area of all existing pulses behaves strictly in accordance with the McCall-Hahn area theorem Eq. (4) and remains close to $2 \pi$. This is realized due to the precise spatial consistency of all the echoes involved.

The case of $\theta_{2}(0)>\pi$ really helps to highlight the benefits of looking at an individual echo signal rather than at the sum of all echo signals. The second incoming pulse is big enough

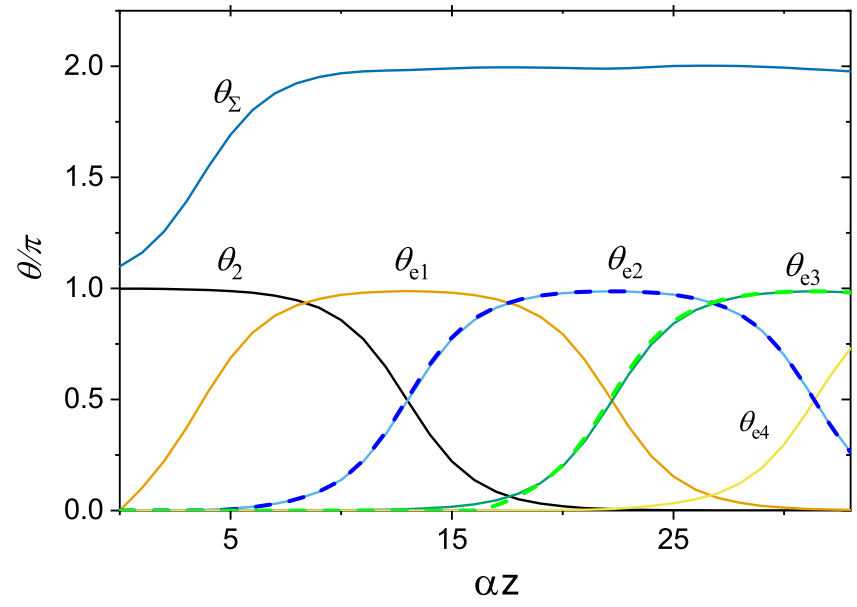

FIG. 2. The multipulse excitation in an optically dense medium. Incoming pulse areas are $\theta_{1}(0)=0.1 \pi, \theta_{2}(0)=0.999 \pi$. The dashed lines show the approximate solution for the second echo $\theta_{e 2}\left(\alpha z_{1}=\right.$ 4.1; blue dashed line $)$ and the third echo $\theta_{e 3}\left(\alpha z_{2}=16.3\right.$; green dashed line).

to form a $2 \pi$ soliton on its own, and the McCall-Hahn area theorem predicts that the sum of all echoes will equal $0 \pi$. The impression could be that after some point in the medium there are no echoes at all. The real picture, however, is much more vivid: there are many hidden echoes with nontrivial areas working together to comply with the McCall-Hahn area theorem. Figure 3 showcases this echo pulse behavior for $\theta_{1}(0)=0.1 \pi, \theta_{2}(0)=1.001 \pi$. Each two of the subsequent echoes have opposite phases, so they are canceling each other in a dynamical equilibrium, resulting in $0 \pi$ total pulse area at any point of the medium. Figure 3 also shows that the primary echo assists the formation of the $2 \pi$ total area, which would otherwise happen much further into the medium.

We note that the echo areas in Figs. 2 and 3 behave very similarly, differing only in their spatial delays. This is the case when we can neglect the stimulated echo terms in Eqs. (8) and find a highly accurate approximate analytic solution for each pulse area. For example, we write for the secondary

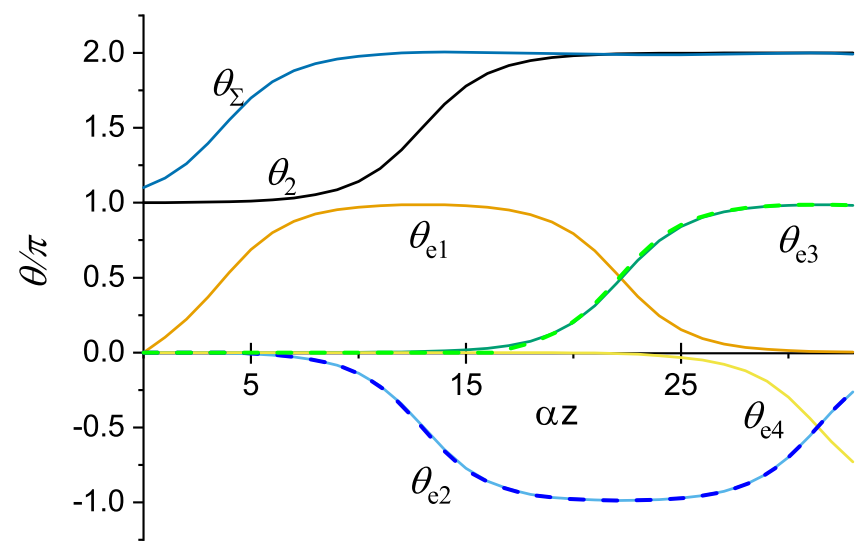

FIG. 3. Evolution of the multipulse excitation in an optically dense medium. Incoming pulse areas are $\theta_{1}(0)=0.1 \pi, \theta_{2}(0)=$ $1.001 \pi$. The dashed lines show the approximate solution for the second echo $\theta_{e 2}\left(\alpha z_{1}=4.1\right.$; blue dashed line $)$ and the third echo $\theta_{e 3}$ ( $\alpha z_{2}=16.3$; green dashed line). 
echo area $\left(z>z_{1}\right)$

$$
\tan \frac{\theta_{e 2}}{2}=\Gamma_{\tau} \sin \theta_{2}\left(z_{1}\right) \sin ^{2} \frac{\theta_{e 1}(z)}{2} \sinh \frac{\alpha}{2}\left(z-z_{1}\right),
$$

where $\theta_{e 1}$ is given in Eq. (7) with the initial pulse areas taken at the transition point $z_{1}:\left(\theta_{1}(0), \theta_{2}(0)\right) \rightarrow\left(\theta_{2}\left(z_{1}\right), \theta_{e 1}\left(z_{1}\right)\right)$. By doing so we assume that at $z=z_{1}$ the first pulse was successfully absorbed by the media and neglect polarization and inversion components acquired at $z<z_{1}$. The solution for $\theta_{e 2}$ is shown with dashed lines in Figs. 2 and 3.

Equations (7) and (9) describe the pulse area at the output of the optically dense media. Moreover, given $\delta t_{1}>\delta t_{2}$ they can also accurately describe the peak energy of the echo pulse $[41,45]$. This easy-to-measure quantity can be used for coherent multipulse spectroscopy of the optically dense media, where the usual spectroscopy is complicated due to strong nonlinear light-atom interaction. In this highly nonlinear regime the conventional Beer law $I_{\text {echo }}=I_{0} \Gamma_{\tau}^{2}$ is not valid while Eq. (7) can be used to measure $\Gamma_{\tau}$ dependence.

It also is interesting to discuss the experimental detection of photon echo train generation and what it can lead to. As seen in Figs. 2 and 3, one can experimentally observe only 2 or 3 light pulses at the output of the optical density medium, while other pulses will be highly suppressed. Thus in media with higher optical densities, we will see only higher order echo pulses, characterized experimentally by later arrival times. The photon echo experiments in such media are quite typical for many quantum memory protocols. In particular, an interesting opportunity is to try detecting the spatial evolution of the photon echo inside such media, for example in the rare-earth ion doped crystals $[14,47,48]$.

One possible candidate for high optical density and large Rabi frequency is the ${ }^{4} I_{9 / 2-}{ }^{4} F_{3 / 2}$ transition of $\mathrm{Nd}^{3+}: \mathrm{YVO}_{4}$ at $897.705 \mathrm{~nm}$ with dipole moment $d=9.16 \times 10^{-32} \mathrm{Cm}$. Considering $P=100 \mathrm{~mW}$ and a beam radius of $r=1 \mu \mathrm{m}$ one could reach up to $\Omega \sim 250 \mathrm{MHz}$. The $\pi$ pulses can be as brief as several nanoseconds, which is much shorter than $T_{2}$. These pulses are spatially squeezed in the medium up to 4 orders of magnitude by the group velocity reduction in the presence of a spectral hole in the optical transition [49]; this would allow us to observe the spatial evolution of the solitons and echo pulses inside the medium.

It is worth noting that only soliton-like pulses can propagate through the medium without changing their temporal form and transferring atoms to their initial state. Accordingly, the photon echo pulses in the generated train will be stretched in time and ultimately overlap with each other deep in the medium forming a single $2 \pi$ soliton in the case of Fig. 2. Similarly the stretching echo pulses will asymptotically form a $0 \pi$ breather, for the case of Fig. 3. In the core of these transformations lies the conservation laws of the MaxwellBloch equations [50].
This concludes the long-lasting derivation of the twopulse photon echo area theorem started over 45 years ago in Refs. [29,36,37], providing an analytic solution for the pulse area of any desired photon echo signal. We showcase the power of the pulse area approach by exploring the rich physics behind the two-pulse echo excitation of an optically dense medium in two previously understudied cases: $\theta_{1}(0)<$ $\pi, \theta_{2}(0) \lesssim(\gtrsim) \pi$. We demonstrate that in both these cases a self-reviving echo train is excited deep in the medium with total pulse area $2 \pi$ in the first case and $0 \pi$ in the second previously unknown case. Thus a slight change in the second pulse area can lead to a dramatic change in the nonlinear multipulse media response: an optical soliton in one case or a soliton followed by a breather in the other case. At the same time the complex spatial dynamic of the total nonlinear media response after the two-pulse excitation is precisely aligned with the general McCall-Hahn area theorem prediction.

The developed pulse area approach might serve as an intensity-independent universal tool for deeper studies of photon echo quantum memory protocols, especially for intensive light pulses. The application of this approach to few-photon and single-photon fields is of particular interest when a few atoms (quantum dots, superconducting qubits) are placed in a high- $Q$ resonator and nanowaveguide with strong nonlinear photon-atom interaction. It was shown in such case that even 4-5 absorbers could be enough to create an inhomogeneous broadening and implement an effective photon echo quantum memory protocol $[27,51]$. This means that interaction of the few-photon field with 4-5 two-level atoms will be highly nonlinear and the pulse area approach could be useful. The case of a single-photon field is special because its consideration in the James-Cumming interaction model is reduced to a system of linear equations. However, even here the application of the area theorem can be relevant, when there are additional intense controlling laser fields. Detailed study of these problems requires special research.

In conclusion, the photon echo pulse area theorem can provide insights in general analysis of coherent multipulse interactions with various photon echo experiments, coherent spectroscopy, and generation of nonlinear waves in optically dense media. It can be used for deeper studies of quantum memory protocols in both optical and microwave wavelength regions, for two- and three-level atomic ensembles with arbitrary transverse and longitudinal relaxation times, etc. The next important analytic step could be to generalize and extend the results acquired here for multipulse excitation using an inverse scattering transform, as was done in Ref. [52] for the McCall-Hahn area theorem.

We thank Joseph Eberly for the stimulating discussion of this work. S.A.M. and R.V.U. acknowledge support from Russian Foundation for Basic Research, Research Project No. 17-52-560009. M.S. gratefully acknowledges Iran National Science Foundation (INSF) for the financial support of this study (Grant No. 96003886).
[1] M. K. Yetzbacher, N. Belabas, K. A. Kitney, and D. M. Jonas, J. Chem. Phys. 126, 044511 (2007).
[2] N. Christensson, B. Dietzek, T. Pascher, A. Yartsev, and T. Pullerits, Chem. Phys. Lett. 457, 106 (2008). 
[3] K. E. Dorfman, F. Schlawin, and S. Mukamel, Rev. Mod. Phys. 88, 045008 (2016).

[4] L. Pezzè, A. Smerzi, M. K. Oberthaler, R. Schmied, and P. Treutlein, Rev. Mod. Phys. 90, 035005 (2018).

[5] G. Mourou, Rev. Mod. Phys. 91, 030501 (2019).

[6] U. K. Kopvillem and V. R. Nagibarov, Fiz. Metal. i Metalloved. 15, 313 (1963).

[7] N. A. Kurnit, I. D. Abella, and S. R. Hartmann, Phys. Rev. Lett. 13, 567 (1964).

[8] C. V. Heer and P. F. McManamon, Opt. Commun. 23, 49 (1977).

[9] V. A. Zuikov, V. V. Samartsev, and R. G. Usmanov, Pis'ma Zh. Eksp. Teor. Fiz. 32, 293 (1980).

[10] E. I. Shtyrkov, V. S. Lobkov, S. A. Moiseev, and N. G. Yarmukhametov, Sov. Phys. JETP 54, 1041 (1981).

[11] T. W. Mossberg, Opt. Lett. 7, 77 (1982).

[12] N. W. Carlson, W. R. Babbitt, and T. W. Mossberg, Opt. Lett. 8, 623 (1983).

[13] S. A. Moiseev and S. Kröll, Phys. Rev. Lett. 87, 173601 (2001).

[14] W. Tittel, M. Afzelius, T. Chaneliére, R. Cone, S. Kröll, S. Moiseev, and M. Sellars, Laser Photonics Rev. 4, 244 (2010).

[15] A. I. Lvovsky, B. C. Sanders, and W. Tittel, Nat. Photonics 3, 706 (2009).

[16] M. Hosseini, B. Sparkes, G. Campbell, P. Lam, and B. Buchler, Nat. Commun. 2, 174 (2011).

[17] I. Usmani, M. Afzelius, H. de Riedmatten, and N. Gisin, Nat. Commun. 1, 12 (2010).

[18] F. Bussières, N. Sangouard, M. Afzelius, H. de Riedmatten, C. Simon, and W. Tittel, J. Mod. Opt. 60, 1519 (2013).

[19] M. Hosseini, G. Campbell, B. M. Sparkes, P. K. Lam, and B. C. Buchler, Nat. Phys. 7, 794 (2011).

[20] M. Rančić, M. P. Hedges, R. L. Ahlefeldt, and M. J. Sellars, Nat. Phys. 14, 50 (2018).

[21] M. M. Minnegaliev, K. I. Gerasimov, R. V. Urmancheev, and S. A. Moiseev, Quantum Electron. 48, 894 (2018).

[22] E. Saglamyurek, T. Hrushevskyi, A. Rastogi, K. Heshami, and L. J. LeBlanc, Nat. Photonics 12, 774 (2018).

[23] J. Guo, X. Feng, P. Yang, Z. Yu, L. Q. Chen, C.-H. Yuan, and W. Zhang, Nat. Commun. 10, 148 (2019).

[24] M. Mazelanik, M. Parniak, A. Leszczyński, M. Lipka, and W. Wasilewski, npj Quantum Inf. 5, 22 (2019).

[25] N. Sangouard, C. Simon, H. de Riedmatten, and N. Gisin, Rev. Mod. Phys. 83, 33 (2011).

[26] H. Wu, R. E. George, J. H. Wesenberg, K. Mølmer, D. I. Schuster, R. J. Schoelkopf, K. M. Itoh, A. Ardavan, J. J. L. Morton, and G. A. D. Briggs, Phys. Rev. Lett. 105, 140503 (2010).
[27] S. A. Moiseev, K. I. Gerasimov, R. R. Latypov, N. S. Perminov, K. V. Petrovnin, and O. N. Sherstyukov, Sci. Rep. 8, 3982 (2018).

[28] S. L. McCall and E. L. Hahn, Phys. Rev. 183, 457 (1969).

[29] G. L. Lamb, Rev. Mod. Phys. 43, 99 (1971).

[30] L. Allen and J. Eberly, Optical Resonance and Two-Level Atoms, Dover Books on Physics and Chemistry (Dover, New York, 1975).

[31] J. Eberly, Opt. Express 2, 173 (1998).

[32] J. H. Eberly and V. V. Kozlov, Phys. Rev. Lett. 88, 243604 (2002).

[33] T. Chanelière, Opt. Express 22, 4423 (2014).

[34] G. Shchedrin, C. O'Brien, Y. Rostovtsev, and M. O. Scully, Phys. Rev. A 92, 063815 (2015).

[35] R. Gutiérrez-Cuevas and J. H. Eberly, Phys. Rev. A 94, 013820 (2016).

[36] E. L. Hahn, N. S. Shiren, and S. L. McCall, Phys. Lett. A 37, 265 (1971).

[37] R. Friedberg and S. Hartmann, Phys. Lett. A 37, 285 (1971).

[38] S. A. Moiseev, Opt. Spectrosc. 62, 180 (1987).

[39] M. Azadeh, C. Sjaarda Cornish, W. R. Babbitt, and L. Tsang, Phys. Rev. A 57, 4662 (1998).

[40] T. Wang, C. Greiner, and T. Mossberg, Opt. Commun. 153, 309 (1998).

[41] T. Wang, C. Greiner, J. R. Bochinski, and T. W. Mossberg, Phys. Rev. A 60, R757 (1999).

[42] J. Ruggiero, J.-L. Le Gouët, C. Simon, and T. Chanelière, Phys. Rev. A 79, 053851 (2009).

[43] C. Li and X.-Y. Yu, Optoelectron. Lett. 6, 152 (2010).

[44] L. Tsang, C. S. Cornish, and W. R. Babbitt, J. Opt. Soc. Am. B 20, 379 (2003).

[45] R. Urmancheev, K. Gerasimov, M. Minnegaliev, T. Chanelière, A. Louchet-Chauvet, and S. Moiseev, Opt. Express 27, 28983 (2019).

[46] See Supplemental Material at http://link.aps.org/supplemental/ 10.1103/PhysRevResearch.2.012026 for the derivation of the general equation for an arbitrary individual echo pulse area.

[47] T. Chanelière, G. Hétet, and N. Sangouard, Adv. At., Mol., Opt. Phys. 67, 77 (2018).

[48] Y.-L. Hua, Z.-Q. Zhou, C.-F. Li, and G.-C. Guo, Chin. Phys. B 27, 020303 (2018).

[49] M. Sabooni, Q. Li, L. Rippe, R. K. Mohan, and S. Kröll, Phys. Rev. Lett. 111, 183602 (2013).

[50] G. L. Lamb, M. O. Scully, and F. A. Hopf, Appl. Opt. 11, 2572 (1972).

[51] N. S. Perminov and S. A. Moiseev, Sci. Rep. 9, 1568 (2019).

[52] D. J. Kaup, Phys. Rev. A 16, 704 (1977). 